\title{
Neurofibromatosis Type 1 with Inoperable, Progressive, Symptomatic Plexiform Neurofibromas
}

National Cancer Institute

\section{Source}

National Cancer Institute. Neurofibromatosis Type 1 with Inoperable, Progressive,

Symptomatic Plexiform Neurofibromas. NCI Thesaurus. Code C143014.

Progressive, symptomatic neurofibromatosis type 1 associated with plexiform

neurofibromas that cannot be removed surgically without risk of substantial morbidity. 